\title{
LOS SERVICIOS ECOSISTÉMICOS DESDE LA PERCEPCIÓN DE LOS PRODUCTORES DE CAFÉ DE BELÉN DE UMBRÍA, RISARALDA, COLOMBIA
}

\author{
ECOSYSTEM SERVICES: PERCEPTIONS FROM THE COFFEE \\ PRODUCERS IN BELÉN DE UMBRÍA, RISARALDA, COLOMBIA
}

\author{
${ }^{1}$ Ángela María Arango, ${ }^{2}$ Miguel Ángel Dossman, \\ ${ }^{3}$ Juliana Muñoz, ${ }^{4}$ Liliana Bueno, ${ }^{5}$ Jhon Jairo Arias, \\ 'Juan Carlos Camargo, 'Juan Martín Maya
}

\author{
1Magister en Ecotecnología, Universidad Tecnológica de Pereira, Pereira, Colombia \\ 2Magister en Ciencias Ambientales, Universidad Tecnológica de Pereira, Pereira, Colombia \\ ${ }^{3,4}$ Estudiante de Doctorado, Universidad Tecnológica de Pereira, Pereira, Colombia \\ ${ }_{5}^{5}$ Magister en Desarrollo Rural, Universidad Tecnológica de Pereira, Pereira, Colombia \\ ${ }^{6} \mathrm{PhD}$ Ciencias Forestales, Universidad Tecnológica de Pereira, Pereira, Colombia \\ 'Esp. en SIG, Universidad Tecnológica de Pereira, Pereira, Colombia

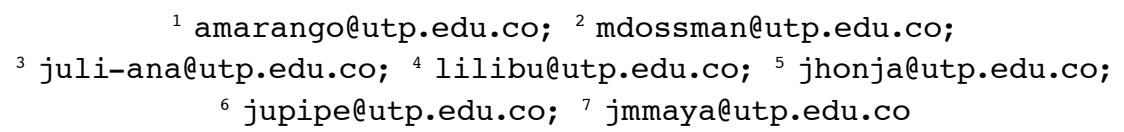

\section{RESUMEN}

Contextualización: los sistemas cafeteros presentes en el departamento de Risaralda, Colombia, se caracterizan por modelos de producción acordes con las condiciones climáticas de la zona, la topografía y las necesidades de los productores de café. Los servicios ecosistémicos son percibidos por las comunidades como los beneficios que se reciben de la naturaleza. Tradicionalmente estos beneficios se asocian con el aumento de la productividad, disfrute de la belleza escénica y recreación, entre otros. En este sentido los miembros de la asociación de café de alta calidad cuchilla de San Juan del municipio de Belén de Umbría, Risaralda Colombia han percibido el cambio en estos beneficios que se traducen en disminución de la productividad, erosión del suelo, mayor incidencia de plagas y enfermedades entre otros. 
Vacío de conocimiento: la determinación de la relevancia de los servicios ecosistémicos visto desde la percepción de los productores ha sido algo poco común en sistemas productivos de café y se convierte en una importante fuente de información para la toma de decisiones de manejo de los sistemas, a través de ello se pueden generar perspectivas más integrales del territorio que abarquen perspectivas ecológicas, económicasy culturales de los sistemas productivos de café.

Propósito del estudio: el objetivo de este estudio consistió en determinar cuáles fueron los servicios ecosistémicos más relevantes para 15 productores cafeteros.

Metodología: se utilizó la metodología de análisis multicriterio de jerarquización y cualificación y talleres con los productores con el fin de recopilar la información.

Resultados y Conclusiones: los resultados mostraron que los servicios de regulación obtuvieron las calificaciones más altas (regulación hídrica y conservación del suelo) seguidos por el servicio ecosistémicos de provisión de alimentos y la disponibilidad de nutrientes (provisión). Por último, se generaron, a través de talleres internos, estrategias de manejo con las cuales se espera que se mantengan los servicios ecosistémicos priorizados por los productores y que permitan un mejor uso y disfrute del medio ambiente.

Palabras clave: Café; Beneficios Ambientales; Funciones de los ecosistemas; Manejo de los sistemas agrícolas; Productores.

\section{ABSTRACT}

Contextualization: In Risaralda, Colombia, coffee production systems have been characterized by production models that follow factors such as climate conditions, topography and producer needs. For the population, ecosystem services are those that come from the nature, which have traditionally been related to an increase in productivity, and a more enjoyable landscape with recreation activities, among other benefits. Members of the High-Quality Coffee Association "Cuchilla de San Juan", from Belen de Umbria, Risaralda, have seen a detriment in said benefits such as low productivity, increase of pests and diseases, among others.

Knowledge gap: The relevance of ecosystem services, seen through the producer's perspective, has rarely been considered in the case of coffee production systems. Determining this relevance becomes a major information source for decision making. This could reveal more comprehensive perspectives in regards of the territory that cover the environmental, economic and cultural spheres.

Purpose: The purpose of this study was to determine the most relevant ecosystem services for 15 coffee producers.

Methodology: multi-criteria analysis for prioritization and qualification; for data collection, workshops with the producers were implemented.

Results and conclusion: The results show that regulation services had the highest qualification (water regulation and soil conservation), followed by supply services (food and nutrients supply). Also, through meetings, management strategies have been development to improve management decisions and improve ecosystems services to coffee production systems.

Keywords: Coffee; Environmental Benefits; Ecosystem functions; Management of agricultural systems; Producers. 


\section{RESUMEN GRÁFICO}

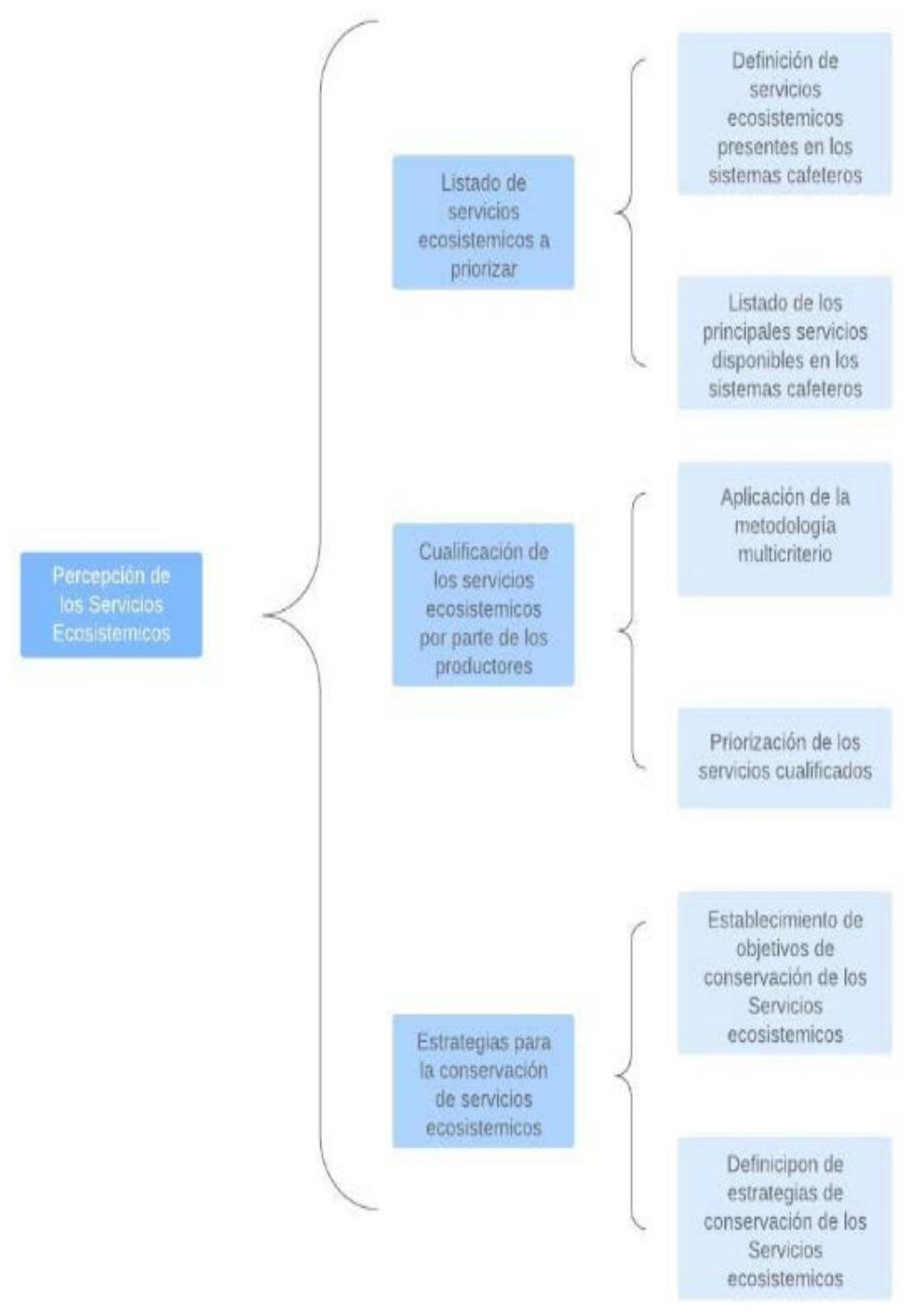

\section{INTRODUCCIÓN}

Colombia es el tercer mayor productor de café en el mundo. Durante el año cafetero $2017 / 2018$ la producción de café mundial alcanzó los 164,8 millones de sacos de café, de los cuales Colombia aportó 13,8 millones (8,4\% del total mundial) (Federación Nacional de Cafeteros y CENICAFÉ, 2018). El departamento de Risaralda por su parte, se encuentra en la denominada zona cafetera de Colombia y la producción de café es la principal actividad económica de la región (Echavarría et al., 2015)
De acuerdo a la Federación Nacional de Cafeteros de Colombia (2018), Risaralda tiene 451mil hectáreas cultivadas con café con un tiempo de establecimiento de 5 años, la densidad promedio de siembra es de 4.103 árboles por hectárea. El municipio de Belén de Umbría contribuye con 8.179 toneladas anuales de café a la producción departamental (Ministerio de Tecnologías de la Información y las Comunicaciones, 2019). Los cultivos de café presentes en el municipio son, en su mayoría, de la modalidad de libre 
exposición. Un estudio desarrollado en 103 fincas pertenecientes a la Asociación de productores de café de Alta Calidad Cuchilla de San Juan demostró que el $41 \%$ de los productores cultivan el café bajo la modalidad de libre exposición (Grupo de Investigacion en Gestión de Agroecosistemas Tropicales Andinos, 2019). En su mayoría, las fincas del municipio cultivan variedades Rosario, CastiIla y Naranjal (Florez R y Arias S, 2017). Estas variedades se caracterizan porque tienen requerimientos nutricionales diferentes, necesitan una mayor exposición al sol y soportan densidades de siembra mayores (Florez $R$ y Arias $S, 2017)$. En términos de producción, las variedades cultivadas han mostrado ser eficientes, sin embargo, su masificación ha generado impactos ambientales negativos que van desde la perdida de la biodiversidad hasta la degradación de los suelos, lo que deriva en una pérdida de los servicios ecosistémicos que brinda la naturaleza (Farfán Valencia, 2017; Montenegro Gómez et al., 2019)

Los servicios ecosistémicos han sido definidos por la evaluación de ecosistemas del milenio como los beneficios que obtiene el ser humano de los ecosistemas (Millennium Ecosystem Assessment, 2005) Una definición alternativa establece que los servicios ecosistémicos son las contribuciones directas o indirectas de los ecosistemas al bienestar humano (De Groot et al., 2010). Ambas definiciones concuerdan en el bienestar que obtienen los seres humanos de los ecosistemas.

Los ecosistemas son el capital natural del que disponen los seres humanos para obtener diversos beneficios (Alvarez et al., 2011), estos tienen la capacidad de resistir a las perturbaciones (capacidad de resiliencia) y generar un flujo de servicios al ser humano a través de las funciones que ellos desarrollan (Alvarez et al., 2011; Martín et al., 2009) . Las interacciones entre la estructura y los procesos ecológicos son las que generan los servicios ecosistémicos (De Groot et al., 2002). De esta manera, para diferenciar las funciones de los servicios, se puede decir que las funciones se generan aunque no exista una demanda, uso o disfrute de ellas, mientras que los servicios son percibidos como tal cuando se utilizan de forma consciente o inconsciente por los seres humanos (De Groot et al., 2002; Pulido Pulido et al., 2019). De esta manera, para la definir un servicio ecosistémico se hace el reconocimiento por parte de un beneficiario de la función relacionada (De Groot et al., 2002).

Los ecosistemas cafeteros proveen diversos servicios ecosistémicos que han sido evaluados por varios autores (Gonzalez Gonzalez y Serna Giraldo, 2018), algunos de ellos como la captura de carbono (Espinoza et al., 2012), la regulación hídrica (Ramírez et al., 2010), (Jaramillo et al., 2011) y aquellos derivados de la biodiversidad (Botero et al., 2014) entre otros.

Utilizando un enfoque basado en actores, se valoraron las percepciones locales de los pobladores con respecto a los servicios ecosistémicos y de acuerdo con su valor de uso y a su nivel de importancia. Para ello, en el presente estudio se utilizó el análisis de jerarquía y calificación, que es un tipo de metodología de análisis multicriterio en la que se analizan los puntos de vista de los expertos respecto a la importancia, en términos cuantitativos, de un parámetro establecido (Mendoza et al., 1999). Esta calificación permitió conocer las percepciones que los actores locales (productores) tienen sobre el territorio y las funciones que se pueden obtener del manejo de los recursos naturales.

El objetivo de la investigación consistió en conocer la percepción de los productores sobre los servicios ecosistémicos presentes en los 
sistemas cafeteros y la importancia que se les da a los mismos, con el fin de generar estrategias para su mantenimiento $\mathrm{y} / \mathrm{o}$ manejo que sirvan como base para la toma de decisiones a nivel predial y local.

\section{MATERIALES Y MÉTODOS Área de estudio}

El trabajo se realizó en el municipio de Belén de Umbría, Risaralda, Colombia, localizado en la subregión II del departamento de Risaralda, sobre la vertiente oriental de la cordillera occidental, con productores de café pertenecientes a la asociación de café de alta calidad Cuchilla de San Juan. Las fincas de los asociados se encuentran distribuidas en rangos altitudinales que van desde los 1.400 hasta los 2.000 m.s.n.m. De los 103 productores pertenecientes a la asociación, se seleccionaron 15 productores teniendo en cuenta la representatividad del rango altitudinal en las fincas, el tamaño de productor que hace referencia al área sembrada en café en la finca con respecto al área total de la misma, y el tipo de productor que indica el manejo que se le da al sistema productivo (Figura 1, Mapa de ubicación de las fincas).

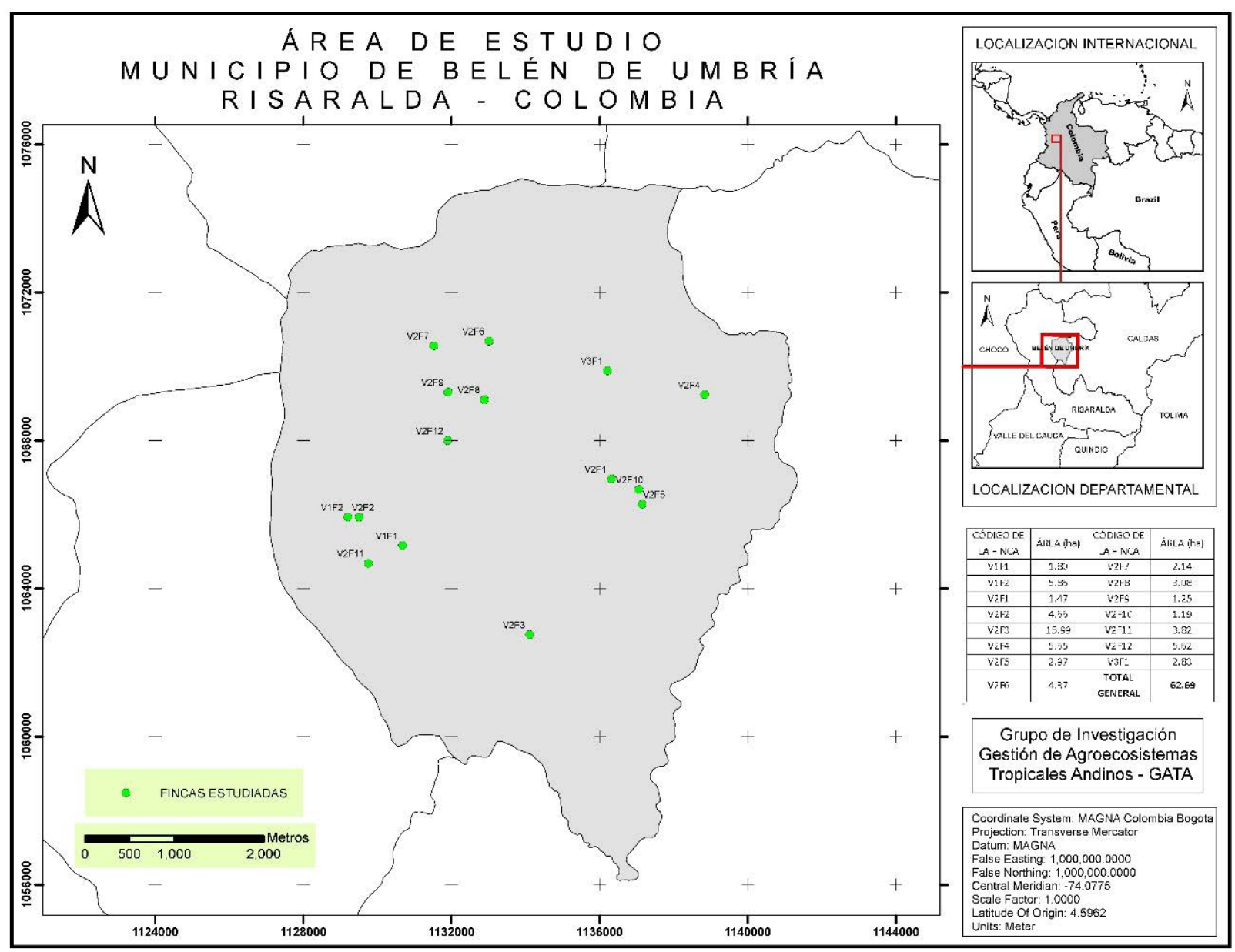

Figura 1. Mapa de ubicación de las fincas.

Fuente: Grupo de Investigacion en Gestión de Agroecosistemas Tropicales Andinos, 2019. 


\section{Percepción de los principales} servicios ecosistémicos asociados

\section{a los agroecosistemas cafeteros}

Para determinar la percepción de los servicios ecosistémicos que proveen los agroecosistemas cafeteros, se utilizó la información recolectada a través del desarrollo del proyecto Servicios Ecosistémicos, en el cual se realizó un muestreo de las propiedades físicas/químicas del suelo y se determinaron los principales servicios ecosistémicos prestados por el suelo (Bedoya y Marín, 2019). De la misma manera, la información recolectada de coberturas del suelo de la zona de estudio a través de las cuales se identificaron las coberturas del agroecosistema (Maya y Camargo, 2019).

Utilizando la información recolectada en los estudios mencionados anteriormente, se realizó una tabla con los principales servicios ecosistémicos que se evaluaron (Tabla 1).

\section{Análisis de jerarquización y calificación}

El análisis de jerarquización y calificación se clasifica dentro de los análisis multicriterio. Esta metodología se utiliza como apoyo para la toma de decisiones durante el proceso de planificación que permite integrar diferentes criterios de acuerdo a la opinión de los actores en un solo marco de análisis para dar una visión integral (Mendoza et al., 1999).

Con el fin de cualificar los servicios ecosistémicos percibidos por los productores, la jerarquización consistió en asignarle una posición a cada elemento de decisión de una lista preestablecida. En este ejercicio se utilizó la jerarquización regular, en la cual, sobre una escala de 1 a 9 (siendo 9 extremadamente importante y 1 débilmente importante), se le asignó a cada servicio un puntaje de acuerdo con el criterio de cada actor. De acuerdo con (Mendoza et al., 1999) en la clasificación regular, diferentes servicios pueden recibir la misma jerarquización, lo que permite cierta flexibilidad en el proceso de la toma de decisiones por parte del productor.

De la misma manera la calificación hace referencia a la asignación de puntuaciones, o porcentajes entre 0 y 100 , de acuerdo con el criterio de un grupo de expertos en el tema, y cuya sumatoria debe ser 100 . Este tipo de calificación recibe el nombre de calificación cardinal, ya que expresa la utilidad o satisfacción que cada experto o productor obtiene al utilizar o consumir en este caso un bien o un servicio ecosistémico (Mendoza et al., 1999; Muñoz et al., 2017).

Para obtener la cualificación final, se sumaron de manera independiente los valores asignados por cada productor tanto de la clasificación como de la calificación (sumatoria valores de clasificación y sumatoria valores de calificación); posteriormente, se asignó un peso relativo a cada atributo con respecto a su valor de importancia $y$, finalmente, a través del peso combinado, se determinó la priorización y cualificación de cada atributo (beneficio).

\section{Estrategias de manejo y conservación de los servicios ecosistémicos}

Después de determinar la priorización de los servicios ecosistémicos, se realizaron talleres internos en los que se definieron estrategias de manejo y conservación, teniendo en cuenta las variables que influyen en la disponibilidad de los servicios ecosistémicos de provisión, regulación, soporte y culturales (Tabla 1).

Con el fin de que las estrategias planteadas respondan a las necesidades de los productores, se clasificaron las fincas en 3 ventanas de acuerdo a su rango de distribución altitudinal: 
Ventana 1: mayores a $1800 \mathrm{msnm}$ - 2 Fincas Ventana 2: entre 1400-1800 msnm - 12 Fincas Ventana 3: menores a 1400 msnm - 1 Finca

\section{RESULTADOS Y DISCUSIÓN Servicios ecosistémicos priorizados para su evaluación}

Los servicios ecosistémicos que se presentaron para ser evaluados, se determinaron de acuerdo a las propiedades físicas/químicas y biológicas del suelo (Bedoya y Marín, 2019); el manejo de los agroecosistemas; y las coberturas presentes en los predios (Maya y Camargo, 2019). Para ello, los servicios ecosistémicos se clasificaron de acuerdo a su categoría y la función que prestan. En la Tabla 1 se resumen los resultados obtenidos.

Tabla 1. Categoría, función y definición de los servicios ecosistémicos.

\section{Categoría}

Servicios ecosistémicos de provisión: Bienes tangibles o materiales que provienen de los ecosistemas con un beneficio directo a las personas.

\begin{tabular}{|l|}
\hline Función Evaluada \\
\hline Provisión de alimentos \\
\hline Recurso medicinal \\
\hline Producción de madera \\
\hline Control de la erosión \\
\hline Control de plagas \\
\hline Conservación del suelo \\
\hline Captura de carbono \\
\hline Regulación hídrica \\
\hline Protección de cultivos \\
\hline Regulación de clima \\
\hline Hábitat de especies \\
\hline Disponibilidad de nutrientes \\
\hline Formación del suelo \\
\hline Generación de biomasa \\
\hline Enraizamiento \\
\hline Belleza Escénica \\
\hline
\end{tabular}

Servicios ecosistémicos de Regulación: procesos complejos mediante los cuales se regulan las condiciones del ambiente en que los seres humanos realizan sus actividades.
Servicios ecosistémicos de Soporte: procesos ecológicos básicos que mantienen y aseguran el mantenimiento adecuado de los ecosistemas permitiendo los flujos de servicios de provisión, de regulación y culturales.
Servicios ecosistémicos Culturales: Beneficios no materiales que las personas obtienen a través del enriquecimiento espiritual, la recreación y la apreciación de la belleza.

Fuente: adaptado de: Organización de las Naciones Unidas para la Alimentación y la Agricultura FAO Ministerio de Ambiente y Desarrollo Sostenible - MADS, 2018.

Como se observa en la Tabla 1 se evaluaron las 4 categorías de servicios ecosistémicos con diferentes funciones que se perciben más fácilmente por los entrevistados; esto contribuyó a que las cualificaciones y calificaciones se hicieran sobre los sistemas cafeteros locales de acuerdo con la percepción de los productores.

\section{Jerarquización y clasificación} de los servicios ecosistémicos

Una vez definidos los servicios ecosistémicos, se priorizaron de acuerdo con la percepción de los 15 productores. Los resultados mostraron que los servicios ecosistémicos mejor valorados fueron los de regulación (regulación 
hídrica y conservación del suelo), seguido por el de provisión de alimentos (soporte) y, por último, la disponibilidad de nutrientes (provisión) (Tabla 2).

Tabla 2. Calificación de los Servicios Ecosistémicos por parte de los productores.

\begin{tabular}{|c|c|c|}
\hline Servicio Ecosistémico & Cualificación & Tipo \\
\hline Regulación hídrica & 7,6 & Regulación \\
\hline Conservación del suelo & 7,4 & Regulación \\
\hline Provisión de alimentos & 7,4 & Provisión \\
\hline Disponibilidad de nutrientes & 7,1 & Provisión \\
\hline Regulación de clima & 6,3 & Regulación \\
\hline Control de la erosión & 6,2 & Regulación \\
\hline Captura de carbono & 6,1 & Soporte \\
\hline Control de plagas & 6,0 & Regulación \\
\hline Hábitat de especies & 6,0 & Soporte \\
\hline Enraizamiento & 5,9 & Soporte \\
\hline Generación de biomasa & 5,9 & Soporte \\
\hline Protección de cultivos & 5,8 & Regulación \\
\hline Formación del suelo & 5,7 & Soporte \\
\hline Belleza Escénica & 4,9 & Cultural \\
\hline Recurso medicinal & 4,5 & Provisión \\
\hline Recursos ornamentales & 3,6 & Provisión \\
\hline Producción de madera & 3,5 & Provisión \\
\hline
\end{tabular}

Fuente: adaptado de: (Organización de las Naciones Unidas para la Alimentación y la Agricultura y "Ministerio de Ambiente y Desarrollo Sostenible, 2018)

De acuerdo con lo expresado por los productores, los servicios de regulación son más evidentes dentro de sus sistemas productivos, el primer servicio asociado a la regulación fue la regulación hídrica, lo anterior puede derivarse de los problemas asociados a la disponibilidad de agua y el estrés hídrico que esto genera en los cultivos.
El periodo bimodal de lluvia - sequia se ha visto afectado por la variabilidad climática. Los productores identificaron que, en los últimos años, las épocas sequía y lluvia se han vuelto más extremas y poco diferenciales entre sí. Lo anterior ha generado mayor las plagas y enfermedades, caída de la flor del café, erosión del suelo entre otras. 
La regulación hídrica ha sido definida como la influencia y la capacidad de los ecosistemas de almacenar o liberar agua regularmente de acuerdo a los periodos lluviosos, el tipo de cobertura y uso del suelo; además, las prácticas de manejo están ligadas a este servicio (Nieto et al., 2015). De acuerdo con lo expresado por los productores, la variedad de café que predomina en los sistemas se deriva de la variedad Colombia (variedad castillo, rosario y supremo) que se caracteriza por plantas de porte bajo, ramas laterales más largas y mayor densidad de siembra por hectárea (Florez R y Arias S, 2017). Según lo anterior, la percepción sobre la importancia de la regulación hídrica reflejada en la evaluación, se ajusta con lo planteado por Jaramillo et al. (2011) quien demostró que la interceptación, percolación y escorrentía de los cafetales sin sombrío es $16 \%$ superior en comparación a sistemas agroforestales con árboles, las características antes mencionadas y su combinación con las pendientes de los sistemas productivos generan los problemas de erosión y perdida de suelos en épocas de lluvia que perciben los productores.

Con respecto al segundo servicio ecosistémico mejor cualificado, es decir, la conservación del suelo, la cualificación de este servicio se asocia a la necesidad de los productores de mantener el suelo en óptimas condiciones para estabilizar o mejorar su productividad. La cualificación alta de un servicio ecosistémico como este, indica que existe una necesidad de intervención en las prácticas de manejo de los sistemas productivos. De la misma manera, el servicio de conservación del suelo influye directamente en la provisión de alimentos (Monsalve et al., 2019), que fue el tercer servicio mejor cualificado por los productores. A pesar de lo anterior, una investigación previa sobre la agrobiodiversidad en las fincas objeto de estudio, demostró que hay pocas especies sembradas (Rios Estrada, 2019) lo que tiene dos connotaciones: la primera con respecto a las condiciones del suelo ya que, según Caro-Caro y Torres-Mora (2015), la diversificación de especies contribuye al mejoramiento de las condiciones del suelo y al mantenimiento del mismo; y la segunda está asociada a la seguridad alimentaria y es que a pesar de que los productores valoraron la provisión de alimentos el índice de diversidad de los agroecosistemas (IDA) fue bajo, según lo evaluado en 13 de las 15 fincas (Rios Estrada, 2019). La percepción que tienen los productores sobre la importancia de la conservación del suelo para garantizar el mantenimiento de los nutrientes coincide con los valores obtenidos por Bedoya y Marín (2019) en el cual, la disponibilidad de nutrientes del suelo en los 15 sistemas productivos evaluados arrojó valores altos.

Los servicios de regulación (regulación del clima, el control de la erosión y el control de plagas) y de soporte (captura de carbono y el hábitat de especies) obtuvieron una valoración intermedia. Para los productores, estos servicios representan aquellos que son percibidos de manera diferente. La regulación del clima, por ejemplo, ha sido asociada a los cambios en los regímenes de lluvia y sequía por los productores durante los últimos tiempos. De la misma manera, el control de la erosión es un servicio muy apreciado 
pues las pendientes de las fincas van desde ligeramente inclinada hasta fuertemente escarpada (Grupo de Investigacion en Gestión de Agroecosistemas Tropicales Andinos, 2019). Los servicios de soporte valorados en la categoría intermedia, como la captura de carbono y el hábitat de especies, fueron así percibidos por los productores debido a las consecuencias que la deficiencia de estos servicios ocasiona en el ambiente. El hábitat de especies se ha visto disminuido por los cambios del clima. De acuerdo con los productores, algunas especies de aves que eran muy comunes en la zona ya no se encuentran o han cambiado su rango de distribución.

Los servicios ecosistémicos cualificados con valores inferiores a 6 mostraron que, a pesar de que son importantes para los productores, su relevancia es menor que los mencionados anteriormente. Los servicios culturales (Mosquera Mena et al., 2019), por ejemplo, no obtuvieron una valoración alta. Lo anterior puede obedecer al hecho de que los productores son oriundos de la zona y están acostumbrados al paisaje.

El servicio ecosistémico que obtuvo menor valoración por los productores correspondió a la producción de madera, la utilización de madera como combustible ha sido reemplazada por fuentes de energía diferentes como gas, o energía eléctrica; los productores cualificaron este servicio más por la extracción de piezas para la construcción que por su utilización como combustible.

\section{Estrategias de conservación} y manejo para la conservación de los servicios ecosistémicos

Las estrategias de conservación y manejo de los servicios ecosistémicos se clasificaron de acuerdo con las ventanas altitudinales establecidas en las cuales se encuentran clasificadas las 15 fincas objeto de estudio. De esta manera se definieron los objetivos a los cuales debería apuntar la estrategia de conservación y manejo de los servicios ecosistémicos para lograr estos objetivos (ver Tabla 3).

Ventana 1: los productores de las fincas ubicadas en la ventana 1 deben conservar los servicios ecosistémicos garantizando la producción de sus fincas; para ello se propone que mejoren la funcionalidad ecosistémica y los flujos de biodiversidad en sus predios, así como la disminución de la deforestación y la presión sobre los bosques.

Ventana 2: este rango altitudinal corresponde al considerado óptimo para el desarrollo de la actividad cafetera, las estrategias para esta ventana se enfocan en la productividad sostenible buscando siempre la conservación de los servicios ecosistémicos prestados por los sistemas productivos de café.

Ventana 3: el rango altitudinal de esta ventana se considera restrictivo, la caficultura presenta problemas por la incidencia de plagas y enfermedades bajo los 1.400 msnm; para las fincas de este rango las estrategias se enfocan en la utilización de insumos alternativos, conservación de los recursos hídricos y mejoramiento de la conectividad. 
Tabla 3. Estrategias de conservación de los servicios ecosistémicos.

Estrategia

Descripción
Categoría de los Servicios ecosistémicos a mejorar

VENTANA 1

\begin{tabular}{l|l|l}
\hline Mejorar funcionalidad ecosistémica & $\begin{array}{l}\text { Mejorar las practicas productivas } \\
\text { de aplicación de agroinsumos, incorporación } \\
\text { de materia orgánica al suelo, diversificación } \\
\text { de especies vegetales }\end{array}$ & \begin{tabular}{c} 
Provisión \\
\hline Mejorar flujos de biodiversidad
\end{tabular} \\
\hline $\begin{array}{l}\text { Contrarrestar dinámicas } \\
\text { de deforestación }\end{array}$ & $\begin{array}{l}\text { Generar/mantener corredores biológicos } \\
\text { no ampliación de la } \\
\text { frontera agrícola. }\end{array}$ & Soporte \\
\hline
\end{tabular}

VENTANA 2

Reducir la emisión de gases de efecto invernadero

Mejorar prácticas de manejo

Aumento de biodiversidad vegetal

Mejorar la conectividad ecosistémica

\section{VENTANA 3}

Mejorar el control biológico

M

Adaptación del sistema productivo

Mantener los recursos hídricos

Utilizar agroinsumos alternativos que no provengan de síntesis química

Conservar coberturas vivas, utilización de herramientas menos agresivas

Siembra de árboles en diferentes arreglos agroforestales

Generar espacios de corredores biológicos
Regulación

Soporte

Soporte

Regulación
Utilizar agroinsumos de origen biológico para prevenir y controlar plagas y enfermedades

Mejoramiento del sistema de productivo de café a través de la diversificación (incorporación de árboles y rotación de cultivos)

Mejorar y mantener las coberturas del suelo para disminuir escorrentía.
Regulación

Soporte

Regulación

Fuente: Autores 
La implementación de las estrategias de conservación y manejo de los servicios ecosistémicos permitirán que los productores mejoren la percepción que tienen sobre los mismos, ya que estos identifican las acciones que aumentan la disponibilidad de cada uno de ellos. En este mismo sentido, se espera que los servicios culturales aumenten a medida que se generen procesos de apropiación del territorio con la implementación de las estrategias.

\section{CONCLUSIONES}

Los servicios ecosistémicos mejor cualificados correspondieron a los de regulación (hídrica, conservación de suelos) los cuales fueron más fácilmente identificados por los productores en el desarrollo de sus actividades diarias en los sistemas de producción.

La disponibilidad de alimentos, cualificada en cuarto lugar, se percibe como los beneficios directos que se obtienen de los sistemas productivos; a pesar de la cualificación del servicio la diversificación no es una constante en los sistemas evaluados.

La metodología de análisis multicriterio con base en la jerarquización y cualificación permite el diseño de estrategias que pueden ser llevadas a cabo para la toma de decisiones dentro de los sistemas productivos.

Los sistemas de producción evaluados a través de la percepción de sus propietarios tienen en común los modelos de producción basados en la rentabilidad y el aumento de la productividad, a pesar de lo anterior los productores son conscientes de los beneficios de la naturaleza (servicios ambientales) y su importancia para la conservación.

\section{CONTRIBUCIÓN DE LA AUTORÍA}

Primer Autor: Investigación, análisis de datos, conceptualización, escritura borrador original y ajustes. Segundo Autor: Investigación, metodología, dirección del proyecto marco de investigación. Tercer Autor: Logística, revisión, investigación, análisis de datos, conceptualización, escritura borrador original.

Cuarto Autor: Logística, revisión y edición, conceptualización. Quinto Autor: Metodología, investigación y análisis de datos. Sexto Autor: edición y revisión del texto final, apoyo en la metodología, investigación y análisis de datos. Séptimo Autor: logística, elaboración de mapas y coberturas.

\section{AGRADECIMIENTOS}

Los autores expresan su agradecimiento al Proyecto "Servicios ecosistémicos, adaptación al cambio climático y planificación del territorio: estrategias para el manejo de sistemas socio ecológicos en la zona cafetera de Colombia" (Código 111074558624 Contrato Nº482017 Financiado por Colciencias), a la Asociación de productores de Alta Calidad Cuchilla de San Juan del Municipio de Belén de Umbría.

\section{LITERATURA CITADA}

Alvarez, P., Ayuso, A. M., De la Cruz, J. L., Guiata, N., Jimenez, L. M., Landa, L., .... Morán, A. (2011). Biodiversidad en españa. base de la sostenibilidad ante el cambio global. Observatorio de la Sostenibilidad en España. Recuperado de https://www. upv.es/contenidos/CAMUNISO/info/U0637187.pdf

Bedoya, B. D., y Marín, J. (2019). Valoración ecológica de los servicios ecosistémicos prestados por el suelo en fincas cafeteras de la Cuchilla de San Juan, municipio de Belén de Umbría, Colombia (Tesis de Pregrado). Universidad Tecnológica de Pereira, Colombia.

Botero, J. E., Lentijo, M. G., y Sánchez, M. L. (2014). Biodiversidad en zonas Cafeteras de Colombia. Avances Técnicos Cenicafé, 444, 12. http://biblioteca.cenicafe.org/bitstream/10778/469/1/ avt0444.pdf

Caro-Caro, C. I., y Torres-Mora, M. A. (2015). Servicios ecosistémicos como soporte para la gestión de sistemas socioecológicos: aplicación en agroecosistemas. Orinoquia, 19(2), 237. https:// doi.org/10.22579/20112629.338 
De Groot, R., Fisher, B., y Christie, M. (2010). Integrating the ecological and economic dimensions in biodiversity and ecosystem service valuation. In P. Kumar (Ed.), The Economics of Ecosystems and Biodiversity: Ecological and Economic Foundations (Issue January, pp. 149-182). Earthscan. https://doi.org/10.4324/9781849775489

De Groot, R., Wilson, M., y Boumans, R. (2002). A typology for the classification, description and valuation of ecosystem functions, goods and services. Ecological Economics, 41(3), 393-408. https://doi.org/10.1016/S0921-8009(02)00089-7

Echavarría, J. J., Esguerra, P., McAllister, D., y Robayo, C. F. (2015). Informe de la misión de estudios para la competitividad de la caficultura en Colombia. Recuperado de https://www.urosario. edu.co/Mision-Cafetera/Archivos/Resumen-Ejecutivo-version-definitiva/

Espinoza, W., Krishnamurthy, L., Vázquez, A., y Torres, A. (2012). Almacén De Carbono En Sistemas Agroforestales Con Café. Revista Chapingo Seria Ciencias Forestales y Del Ambiente, XVIII(1), 57-70. http://dx.doi.org/10.5154/r. rchscfa.2011.04.030

Farfán, F. (2017). Sistemas de producción con café a libre exposición solar o en sistemas agroforestales Condiciones para su establecimiento. Avances Técnicos Cenicafé, 851, 839-851.

Federación Nacional de Cafeteros, y CENICAFÉ. (2018). Informe Anual Federación Nacional de Cafeteros - CENICAFÉ (P. Benavides, J. Ramos, E. Góngora, J. Acuña, y M. Diana (eds.)).

Florez R, C. P., y Arias S, J. C. (2017). Guía para la caracterización de las variedades de café: Claves para su identificación. Avances Técnicos Cenicafé, 476, 1-12.

Gonzalez Gonzalez, M., y Serna Giraldo, C. A. (2018). Servicios ecosistemicos potenciales en el sector cafetero Colombiano. Cenicafe, 69(2), 34-46.

Grupo de Investigacion en Gestión de Agroecosistemas Tropicales Andinos. (2019). Informe Preliminar de resultados del proyecto Servicios ecosistémicos, Adaptación al Cambio Climático y Planificación del Territorio: Estrategias para el manejo de Sistemas Socioecológicos en la zona Cafetera de Colombia.

Jaramillo, Á., Ramírez, V., y Arcila, J. (2011). Distribución de la lluvia clave para planificar las labores en el cultivo del café en Colombia. Avances Técnicos Cenicafé, 411, 1-8.

Martín, B., Gómez, E., y Montes, C. (2009). Un marco conceptual para la gestión de las interacciones naturaleza-sociedad en un mundo cambiante. Cuides - Cuaderno Interdisciplinar de Desarrollo Sostenible, 3, 229-258.

Maya, J. M., y Camargo, J. C. (2019). Sensoramiento remoto y vehículos aéreos no tripulados (VANT) para la caracterización de agroecosistemas cafeteros / Servicios ecosistémicos, adaptación al cambio climático y planificación del territorio: Estrategias para el manejo de Sistemas Socioecológicos en la zona Cafetera de Colombia.

Mendoza, G. A., Macoun, P., Prabhu, R., Sukadri, D., Purnomo, H., y Hartanto, H. (1999). Guidelines for applying multi-criteria analysis to the assessment of criteria and indicators. In Guidelines for applying multi-criteria analysis to the assessment of criteria and indicators. Center for International Forestry Research - CIFOR. https:// doi.org/10.17528/cifor/000769

Millennium Ecosystem Assessment, 2005. Ecosystems and Human Well-being: Biodiversity Synthesis. World Resources Institute, Washington, DC.

Ministerio de Tecnologías de la Información y las Comunicaciones. (2019). Plataforma de Datos Abiertos de Colombia. https://www.datos.gov.co/

Monsalve, L. M., Valencia, F. L., Guzmán, A. R., Duque, C. M., Pérez, D. A., Valderrama , .... Polanco, M. F. (2019). Servicio ecosistémico de abastecimiento: alimentos. En Servicios ecosistémicos: un enfoque introductorio con experiencias del occidente Colombiano, Cap. 2, pp. 35-56. https:// doi.org/10.22490/9789586516358.02

Montenegro, S. P., Barrera, S. E., Chiriví, J. S., Pulido, S. Y., Sepúlveda, Y. A., Vinasco, M. C., y Palomino Leiva, M. L. (2019). Prevención de la erosión y conservación de la fertilidad del suelo. En Servicios ecosistémicos: un enfoque introductorio con experiencias del occidente Colombiano, Cap. 9. 172-187. https://doi. org/10.22490/9789586516358.09

Mosquera Mena, R. A., Carmona Cadavid, M. E., Pulido Pulido, S. Y., Vinasco Guzmán, M. C., Moraes Boldini, J., Barrera Berdugo, S. E., y Montenegro Gómez, S. P. (2019). Recursos Medicinales. En Servicios ecosistémicos: un 
enfoque introductorio con experiencias del occidente Colombiano, Cap. 4. 172-187. https://doi. org/10.22490/9789586516358.04

Muñoz, J., Camargo, J. C., y Romero, C. (2017). Beneficios de los bosques de guadua como una aproximación a la valoración de servicios ecosistémicos desde la "Jerarquización y Calificación". Gestión y Ambiente, 20(2), 222-231. https://doi. org/10.15446/ga.v20n2.66603

Nieto, M., Cardona, L. y Agudelo, C. (2015). Análisis de servicios ecosistémicos. Provisión y regulaciónhídrica. En: Ungar, P. (ed.) (2015). Hojas de ruta. Guías para el estudio socioecológico de la alta montañaen Colombia. Bogotá: Instituto de Investigación de Recursos Biológicos Alexander von Humboldt. http://hdl.handle. net/20.500.11761/9296

Organización de las Naciones Unidas para la Alimentación y la Agricultura, y Ministerio de Ambiente y Desarrollo Sostenible. (2018). Guía de buenas la gestión y uso prácticas para de los suelos sostenible en áreas rurales. Organización de las Naciones Unidas para la Alimentación y la Agricultura, Ministerio de Ambiente y Desarrollo Sostenible.
Pulido, S. Y., Mosquera, R. A., Rodríguez, S. A., Fonseca, M. R., Montenegro, S. P., Valencia, F. L., y Barrera, S. E. (2019). Hábitat para especies. En Servicios ecosistémicos: un enfoque introductorio con experiencias del occidente Colombiano, Cap. 12. 172-187. https://doi. org/10.22490/9789586516358.12

Ramírez, V., Jaramillo, Á., y Arcila, J. (2010). Índices Para Evaluar El Estado Hídrico En Los Cafetales. Cenicafé, 61(1), 55-66.

Rios Estrada, S. K. (2/019). Aproximación metodológica para la valoración ecológica de servicios ecosistémicos generados por la agrobiodiversidad en sistemas productivos cafeteros en el municipio de Belén de Umbría (Tesis de Pregrado). Universidad Tecnológica de Pereira, Colombia. 